\title{
A Historical Perspective on Measurements of Solar Irradiance
}

\author{
By V. G A I Z A US K A S \\ Herzberg Institute of Astrophysics, National Research Council of Canada, \\ Ottawa, Canada K1A OR6
}

Recent measurements made from platforms in space prove beyond question that the radiant energy received from the Sun at the Earth, once called the 'solar constant', fluctuates over a wide range of amplitudes and time scales. The source of that variability and its impact on our terrestrial environment pose major challenges for modern science. We are confronted with a tangled web of facts which requires the combined ingenuity of solar, stellar, planetary and atmospheric scientists to unravel. This brief overview draws attention to key developments during the past century which shaped our concepts about sources of solar variability and their connection with solar activity.

Commission 10 of the International Astronomical Union (IAU) has a long and distinguished association with the subject of this conference on "The Sun as a Variable Star: Solar and Stellar Irradiance Variations". At the time of the first General Assembly of the IAU, held in Rome in 1922, Commission 10 was responsible for 'Solar Radiation'. Its President was Charles Greeley Abbot of the Smithsonian Astrophysical Observatory (Abbot 1922). He is still renowned for directing an epic series of daily measurements of the 'solar constant' from several excellent mountain-top sites for about 30 years. In those early days, the goal of the Commission was to validate the measured variability in the 'solar constant' in terms of solar activity. Subsequent Transactions of the IAU reflect Abbot's vexation with the magnitude of any real variation in the Sun's output. His report to the 1925 General Assembly strikes a plaintive note: "Solar variations exceeding 3\% occur rarely, but evidence is accumulating in yet unpublished meteorological researches that solar changes of as little as $1 / 2$ of one percent are not negligible. Hence the problem of securing satisfying accuracy in solar constant measurements is exceedingly difficult, considering that we must observe at the bottom of a turbid atmospheric sea" (Abbot 1925).

From the IAU's beginnings, the motivation behind the quest for variations in solar luminosity was as much to establish a link with variations in terrestrial climate as with solar activity. The Sun's internal source of energy did not enter into the reports of the solar commissions in the 1920's; the great work on the internal constitution of the stars was just then underway. Not until the next decade would nuclear fusion be proposed as the means of energy generation. And it would take four decades before models for stellar evolution were placed on a secure foundation. Those great conceptual advances would have been impossible without accurate measurements of the luminous outputs of the Sun and of a myriad of stars.

Nuclear fusion in stars leads to their variability in luminosity on time scales determined by the exhaustion of nuclear fuels at each stage in the process for converting mass into radiant energy. These time scales are many orders of magnitude greater than the period of sunspot activity. The awesome panoply of coronal and chromospheric activity that we can witness almost every day represents just a minuscule loss from the Sun's vast reserves of energy. 
And so, by the time they where in a position to measure solar irradiance directly with instruments placed above Abbot's 'turbid sea', solar scientists realized that any variations must be extremely small. Some even argued that there might be no variation at all in the total luminous output. Whatever radiation was blocked by sunspots might reappear elsewhere in order to preserve a perfect balance - as a brightening of the photosphere around spots, as enhanced emission in faculae, or as waves dissipating energy in the corona. These arguments were supported by lingering suspicions about the validity of Abbot's correlations made into the 1950's between measured changes in the 'solar constant' and weather patterns. Recognition also came in the 1950's and 1960's that solar emissions from UV to X-ray wavelengths were far in excess of black-body values extrapolated from photospheric wavelengths and were, besides, highly variable.

These considerations no doubt weighed heavily in the design of the self-calibrating cavity radiometers flown on the Nimbus-7 satellite (ERB experiment, Hickey et al. 1980) and on the Solar Maximum Mission (SMM) satellite (ACRIM experiment, Willson 1981). For the first time, the sensitivity for measuring the total solar irradiance was sufficiently high that dips caused by spots and peaks caused by facular excesses were easily detected and compared successfully with models. Stability of the ACRIM instrument was such that intensity fluctuations due to the 5-minute p-mode oscillations were also recognized. All doubt about the ability of sunspots to block energy flow through the photosphere has been removed; no evidence has since been found to suggest that the contribution of faculae to the solar luminosity cancels that of sunspots. The most important discovery of the Nimbus-7/ERB and SMM/ACRIM irradiance observations was the long-term variation of total solar irradiance over the solar cycle with an amplitude of $0.1 \%$ (Willson \& Hudson 1988). The trend in the variation of the luminosity suggests that it is directly correlated with the population of active regions and the bright magnetic network. Thus one of the long-term goals set out by the original Commission 10 over 70 years ago has been achieved.

But there is no shortage of remaining problems. All one has to do is to glance at the topics covered in this conference. For example, even after the long-term trend in the irradiance values from ACRIM have been corrected for the sunspot deficits and the facular excesses in active regions, an additional irradiance component remains with a solar-cycle time scale. We tend to forget that, even when there are no sunspots at the minimum phase of the solar cycle, the total magnetic flux of the Sun is still about one-quarter of its mean value at the peak of the cycle and is still variable on a small scale.

We are still far from resolving problems related to atmospheric physics in the presence of a variable solar output at UV wavelengths. These problems have ceased to be merely academic. Public concern about the varying distribution of ozone in Earth's middle atmosphere is high and growing. To assess the effect of variable UV output from the Sun, we require precise solar UV spectrophotometry at the $1 \%$ level. In order to assess longterm trends in global warming, more research is needed into the fossil records preserved in ancient sediments and glacial deposits. When, for how long, and by how much did solar activity change significantly from the levels we experience today?

And we must turn to the study of other stars like the Sun to give us insight to the Sun's past and future behavior. It has been pointed out that the ACRIM record shows the total luminosity of the Sun increasing with sunspot activity; yet younger Sun-like stars seem to be anti-correlated with their activity as measured by chromospheric outputs. On the other hand the same stars over a stellar rotation period show an anti-correlation between chromospheric and photospheric outputs. It would seem that the Sun has evolved from an early stage, where its variability was determined by dark spots, to the present one, 
where it is dominated by faculae. Conversely, problems of general interest to stellar evolution can be answered from solar studies of those phenomena which we now know to be responsible for the variability in the Sun's radiative output.

There is much to celebrate in recent achievements in research on solar-stellar variability. But it is no time to rest on laurels. All the topics I have skimmed over rely on access to data banks of uniformly high quality and high precision. Many of the exciting problems uncovered by recent research demand long-term continuity for key data bases. Yet economic realities are threatening the viability of the kind of long-term research discussed in these proceedings. Commitment is now very difficult to extract from funding agencies for programs of synoptic measurements made either in space or at ground level. Given the public awareness about global warming and ozone depletion, we have to be vigorous in promoting those areas of solar research which address those concerns. We have to take great care that key data bases continue to accumulate. As President of Commission $10 \mathrm{I}$ welcome any resolutions on these matters from this meeting to forward to the Executive Committee of the IAU for formal adoption at next year's General Assembly. And I invite you to consider if a new working group, as proposed by the Scientific Organizing Committee of this Colloquium, is needed in the forthcoming reorganization of the solar commissions of the IAU in order to focus attention on these concerns and to propose courses of action.

\section{REFERENCES}

Aввот, C. G. 1922 Trans. Int. Astron. Union 1, 28-30.

Аввот, C. G. 1925 Trans. Int. Astron. Union 2, 27-39.

Hickey, J. R., Stowe, L.L., Jacobowitz, H., Pellegrino, P., Maschhoff, R. H., House, F. \& VONDER HAAR, T. H. 1980 Initial solar irradiance determination from Nimbus-7 cavity radiometer measurements. Science 208, 281-282.

RISE: 1990 Radiative Inputs of the Sun to Earth. NSF Research Plan, Washington.

WILLSON, R. C. 1981 Solar total irradiance observations by active cavity radiometers. Science 74, 217-229.

Willson, R. C. \& Hudson, H. S. 1988 Solar luminosity variations in solar cycle 21. Nature 332, 810-813. 